\title{
Tests with a Hybrid Bearing for a Flywheel Energy Storage System
}

\author{
G. G. Sotelo*1, E. Rodrigues ${ }^{2}$, F. S. Costa ${ }^{2}$, J. G. Oliveira ${ }^{3}$, J. \\ de Santiago $^{4}$, R M Stephan ${ }^{2}$ \\ ${ }^{1}$ Department of Electrical Engineering, Fluminense Federal University, Niterói, Rio \\ de Janeiro, Brazil \\ ${ }^{2}$ Department of Electrical Engineering, Rio de Janeiro Federal University, Rio de \\ Janeiro, Rio de Janeiro, Brazil \\ ${ }^{3}$ Universidade Federal de Juiz de Fora, Cidade Universitaria Plataforma 5 Martelos, \\ CEP 36036-900, Juiz de Fora MG, Brazil \\ ${ }^{4}$ Uppsala University, Ångström Laboratory, 751 21, Uppsala, Sweden \\ E-mail: Corresponding author:gsotelo@id.uff.br
}

\begin{abstract}
This paper describes the design and experimental test of a passive magnetic bearing system composed by a Superconductor Magnetic Bearing (SMB) and a Permanent Magnet Bearing (PMB). This bearing setup is part of a flywheel energy storage system. The advantage of using a passive bearing system is that it offers low friction without the need of a magnetic bearing controller, increasing the reliability and decreasing the energy consumption. The first set of tests were quasi-static radial and axial force measurements of the PMB operating alone and together with the SMB. As the PMB is intrinsically unstable in one degree of freedom, the operation of the SMB together with the PMB is necessary to stabilize the system. After that, dynamic measurements were made for the SMB operating alone and together with the PMB. The resonant speeds were identified and the bearing radial and axial forces were also measured for the SMB and SMB + PMB operation. These results indicate that the studied bearing set is technologically feasible to be used in flywheel energy storage systems.
\end{abstract}

Keywords: Superconducting Bearing, Permanent Magnet Bearing, FESS.

\section{Introduction}

The generation, transmission and distribution of electrical energy changed the industrial sector in the last century. Nevertheless, electrical energy storage is still a challenge. There are several options, such as: batteries [1], fuel cells [2], supercapacitor [3], SMES (Superconducting Magnetic Energy Storage) [4], FESS (Flywheel Enegy Storage System) [5], thermal energy [6], compressed air [7], pumped hydro [8], etc. The literature presents several papers comparing the different energy storage technologies $[9,10,11]$, each one having its specific application niche. Energy storage systems are 
classified according to their energy capacity, power rate, lifetime, availability factor, etc. There is no dominant technology in the specic case of small and medium applications, in the range of few square meters and less of one tonne. The focus of this paper is set in this area.

Figure 1 presents a qualitative diagram of Power Density $\times$ Energy Density of some light and small volume technologies for energy storage. It can be noted that the FESS energy density level in the same magnitude of the batteries, but with higher power density and therefore it has a great potential for different applications. FESS have been studied for decades [12], but they became popular when the technology was introduced into the Formula 1 cars during 2009 [13].

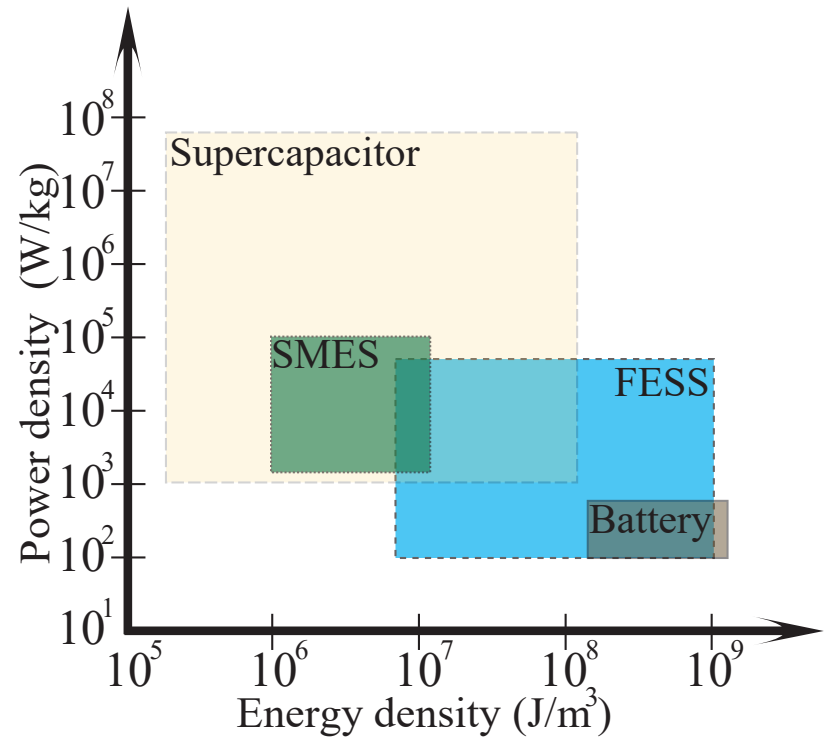

Figure 1. Energy vs. power densities for several technologies of energy storage.

FESS are an environmentally friendly storage solution since no hazardous materials are used in their construction, resulting in a small environmental impact. Flywheels can be applied to several niches, such as: electric grid frequency regulation [14] and load leveling for integration of renewable energy sources, as solar and wind power [15]. The mechanical energy of its rotation is given by:

$$
E=\frac{I \omega^{2}}{2}
$$

where $I$ is the flywheel moment of inertia and $\omega$ is its rotational speed. There are two types of flywheels: heavy with low rotational speed FESS and light with high rotational speed FESS. Light and high speed FESS present higher power and energy densities and they are the focus of our research.

The operation at high speed increases energy density but also the friction losses in the bearings. Being so, it is necessary to find an alternative to replace the traditional rolling bearings, which can be done by the use of magnetic bearings. Magnetic bearings 
can be classified into two major types: active magnetic bearing and passive magnetic bearing. In the literature it is possible to find flywheels assembled with: mechanical bearings [16], hybrid ceramic/magnetic bearings [17], active magnetic bearings [18], and with Superconducting Magnetic Bearings (SMB) $[5,19,20]$. The active technology requires accurate control of the current through the windings of electromagnets and has already some industrial suppliers. An alternative passive magnet bearing set is composed by a SMB and a Permanent Magnet Bearing (PMB). The SMBs present different properties than rolling bearings and active bearings. They have lower force density and require broader air gaps. SMBs present limitations concerning the weight of the flywheel and the air gap required to motor/generator. The main advantage of the PMB and SMB bearing set in relation with the active bearings is the low level of energy consumption and reliability.

Since a completely passive PMB is not stable (as well known by Earnshaw Theorem), and a bearing system using only SMB would be more expensive to build and operate, the hybrid PMB-SMB configuration presents a good benefit cost relation. In other words, the use of SMB allows the vertical stabilization of the PMB for the bearing operation, while the SMB reduces the number of superconductors used into the bearing and the power necessary to cooling the HTS material.

Flywheels with PMB and SMB bearings operating together were studied previously by the group [14]. Prototypes of flywheels with PMB and SMB were also constructed by other authors $[21,22]$ with some differences in the bearing topology (like a radial SMB), proving the technology feasibility. Here, a new PMB and SMB hybrid bearing set was constructed to study the system dynamics. An illustration of the FESS concept, including the bearing set (PMB and SMB), the electric motor/generator and inertial mass is presented in Figure 2.

The SMB can be arranged in rotational [5] or in linear topologies [23] generally designed for MagLev vehicles. The operational principle is the same, changing only the movement direction. In the proposed bearing set, the SMB is essential to stabilize the PMB. The SMB is composed of two parts: a PM array responsible to produce the external field and High Temperature Superconductors (HTS). When a HTS is in the presence of the field produced by the PM array, it will react to create a free rotational movement without friction losses and it will have a stable levitation in all degrees of freedom. In the designed prototype, the SMB is positioned at the bottom part of the FESS, which is responsible to produce vertical and radial forces and stabilize the shaft at the central position.

In previous works of the group $[24,25]$, the levitation force was quasi-statically measured for the SMB operating alone, in other words, without the auxiliary PMB. Some preliminary studies of the SMB and PMB dynamics were presented previously $[26,27]$. In the present paper, a deeper investigation of the bearing set for a flywheel system is done. The static measurements of the PMB and the SMB working separately and together are presented here. From these results the operation position of the bearing set could be determined, and the dynamic operational experiments were done. 


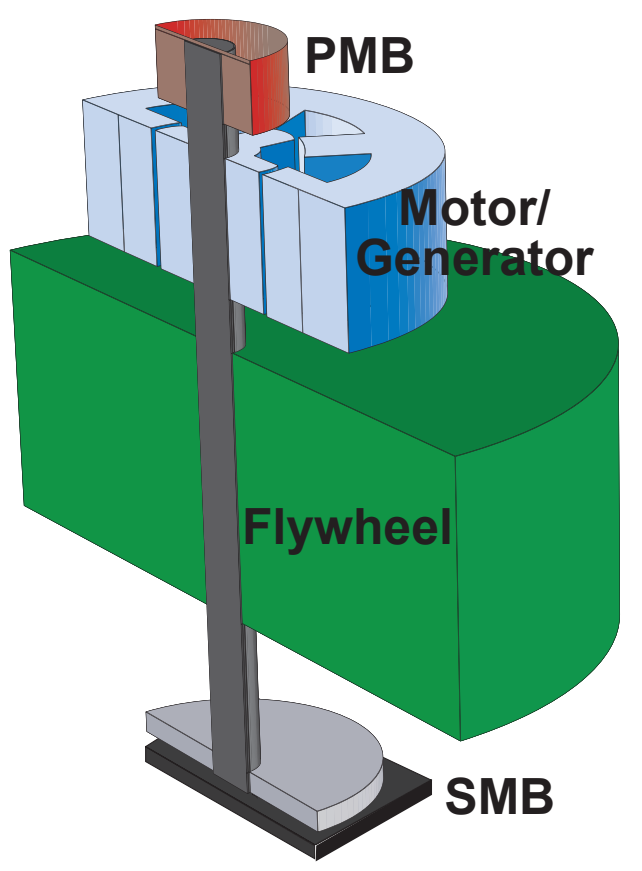

Figure 2. Schematic of the FESS under development.

\section{Methodology}

\subsection{Magnetic Bearing Set}

The experimental set up for the magnetic bearing tests is presented schematically in Figure 3. The focus of these tests is to obtain the magnetic forces. The bearings are tested separately so that the contribution of each bearing is identified. The flywheel shaft and motor presented in Figure 3 are not included in the test. Details of the bearings design are presented in the sequence.

\subsection{Permanent Magnet Bearing}

The upper bearing is a permanent magnet one, and it consists of two rings of axially oriented permanent magnets. This bearing has a very simple construction, since it is composed by two concentric permanent magnet rings, as presented in Figure 4 and Table 1. Some experimental and simulated preliminary results of this PMB, studied without the SMB, were presented in [26, 27]. Some experiments of the static and dynamic tests to characterize the lower passive magnetic bearing will be reported in the results section.

\subsection{Superconducting Magnetic Bearing}

High Temperature Superconductors (HTS) need to be cooled under its critical temperature to present the diamagnetic effect. Also, HTS bulks are doped with impurities to avoid movement of the pined flux. In the SMB, the superconductor bulk 


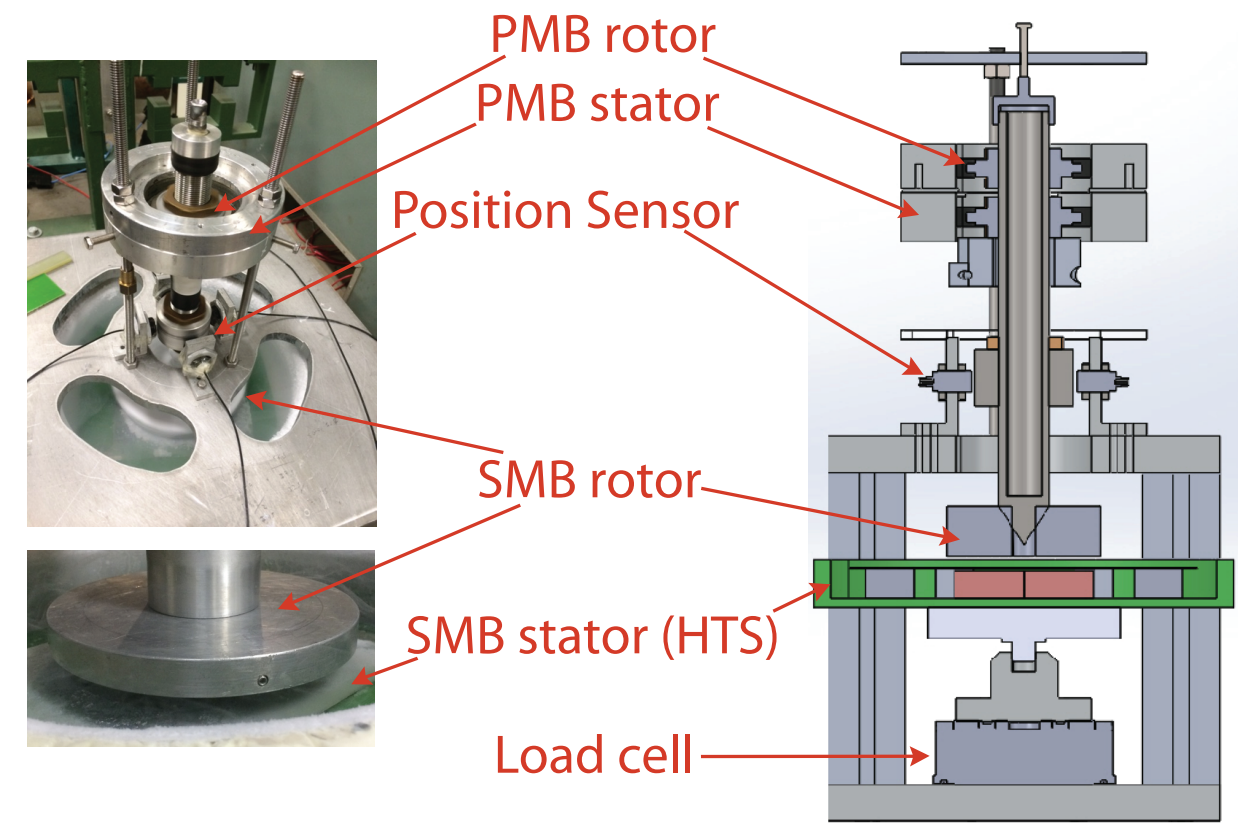

Figure 3. Magnetic bearings test bench.

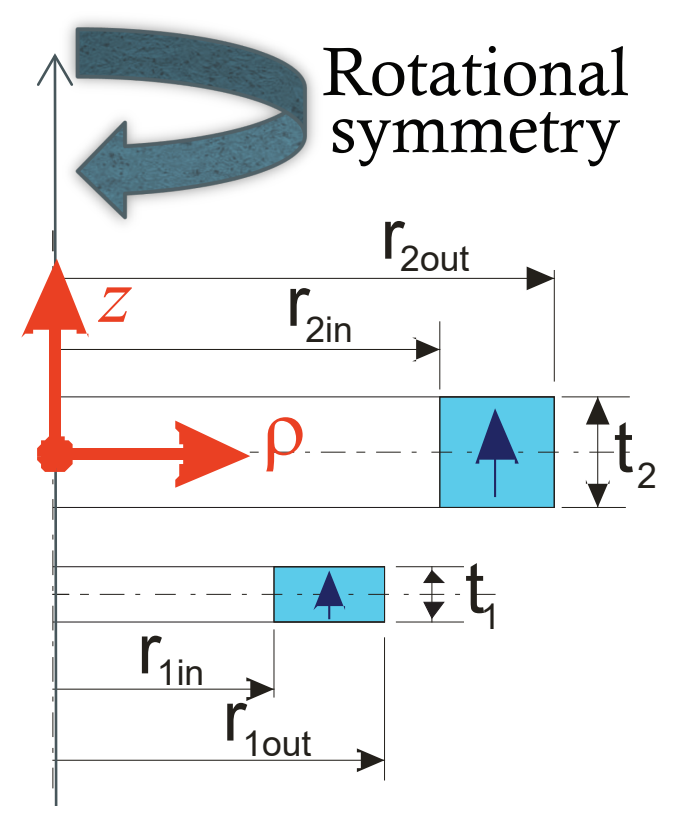

Figure 4. Schematic of the PMB designed. The inner permanent magnet is the rotor and the outer one is the stator.

cooling can be done by a cryocooler system or by a bath in liquid nitrogen $L N_{2}$. Here $L N_{2}$ was used to cool down the HTS bulks in these experiments. The cooling position has an important role for the operation of the SMB, since it will be responsible to guarantee the stability of the bearing by the pined flux. When the HTS is cooled without any external magnetic flux, the procedure is knows as zero field cooling (ZFC). In the case of the SMB, the maximum axial force can be obtained, but the bearing has no radial 
Table 1. Permanent magnet bearing parameters.

\begin{tabular}{|c|c|c|}
\hline Parameters & Description & Length $(\mathrm{mm})$ \\
\hline$r_{1_{\text {in }}}$ & Inner radius of inner ring & 20 \\
$r_{1_{\text {out }}}$ & Outer radius of inner ring & 30 \\
$t_{1}$ & Thickness of inner ring & 5 \\
$r_{2_{\text {in }}}$ & Inner radius of outer ring & 35 \\
$r_{2_{\text {out }}}$ & Outer radius of outer ring & 45 \\
$t_{2}$ & Thickness of outer ring & 10 \\
$S$ & Axial distance from geometric center of each magnet & - \\
\hline
\end{tabular}

stability. This measurement is important to determine the maximum levitation force that the SMB can produce. In the other hand, when the HTS is cooled in the presence of the field produced by the magnetic rotor, in a process named as field cooling (FC), the axial force obtained is smaller than in the ZFC case, but this bearing presents now a radial restoring force and stable levitation.

The SMB prototype is composed by a magnetic rotor in a flux shaper configuration (Figure 5-a and b) $[24,25]$ and eight $\mathrm{Y}_{1} \mathrm{Ba}_{2} \mathrm{Cu}_{3} \mathrm{O}_{7-\delta}$ superconductor (YBCO) bulks enclosed in a static cryostat (Figure 5-c). The dimensions of each YBCO bulk are $67 \times 32 \times 14 \mathrm{~mm}^{3}$.

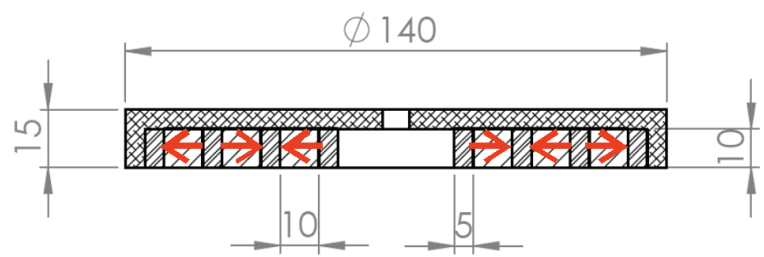

(a)

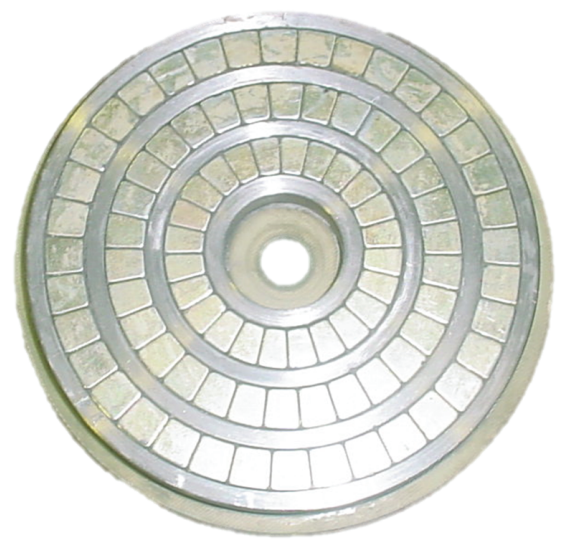

(b)

Figure 5. SMB developed: (a) Dimensions of the SMB rotor (the arrows indicates the magnetization); (b) Photo of the SMB rotor with permanent magnets and iron rings. 


\subsection{Experimental Procedures and Test Bench}

In order to measure the behaviour of the magnetic bearings, two experimental set ups were designed. The test benches were constructed to obtain the quasi-static and dynamic characteristic of the PMB and SMB operating alone and together. For both tests a 6-axes load cell was used. It is able to measure forces and torques along three perpendicular directions. An acquisition board was used to control the system and to acquire the signal from the load cell. A description of each system is presented bellow.

\subsubsection{Quasi-Static Measurement System}

The first measurement system was designed to register the quasi-static forces of the bearings. Figure 6 presents the test bench assembled with the SMB to apply a movement along the radial $(x)$ and axial $(z)$ directions and measure the respective forces. This system has two linear actuators, whose position is controlled by step motors. The system was programmed to proceed the measurements applying discrete displacements of $1 \mathrm{~mm}$ along the desired direction and, after the movement is completed, the system is stopped during 1 second to acquire the forces. The mean value of the force along this time is considered as the result for the respective position. This procedure is done in order to let the transitory HTS flux creep effects pass. After that, other position step is applied and the force is measured at the new position. This procedure is repeated for all the desired positions.

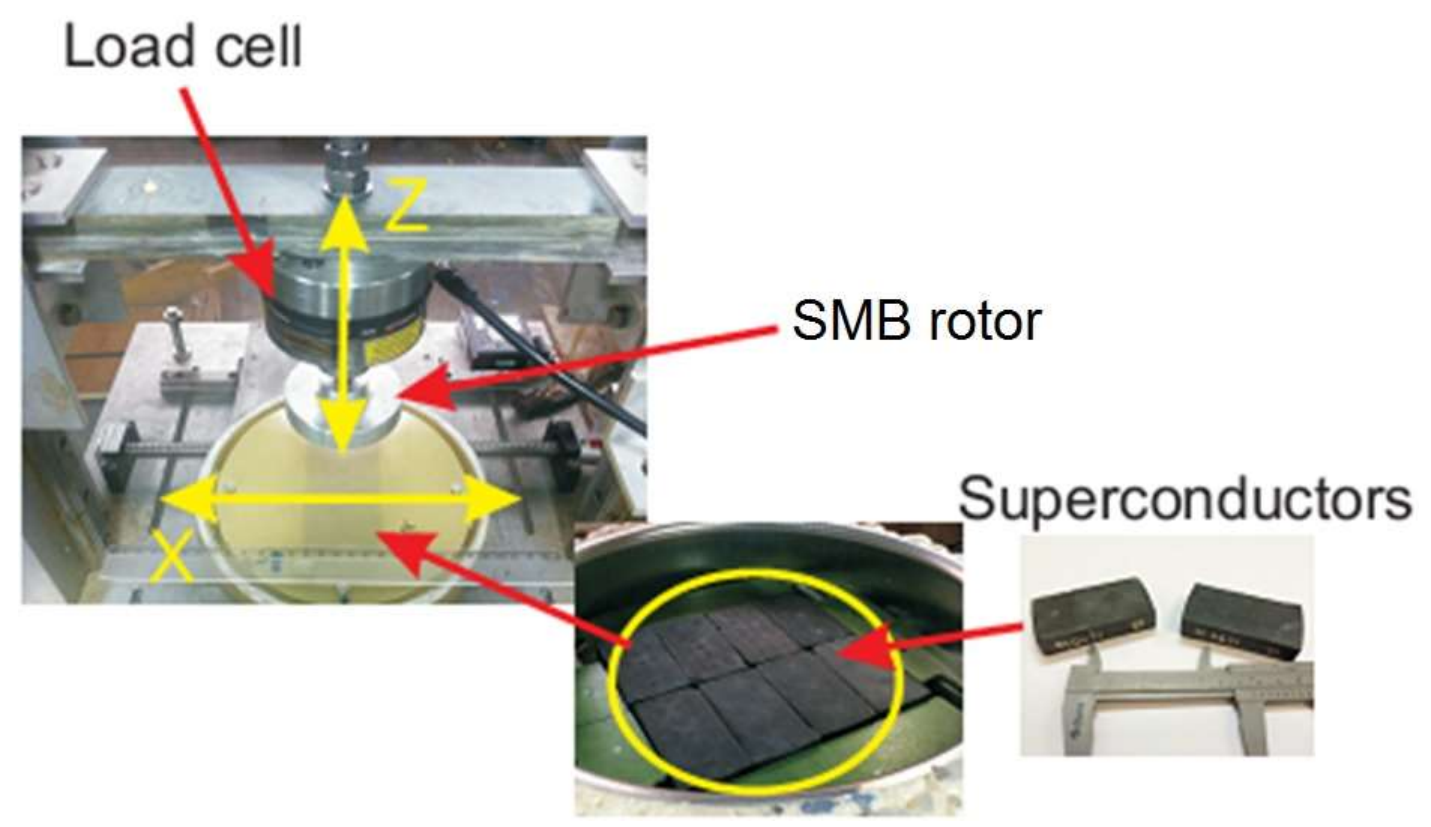

Figure 6. Test bench for the quasi-static measurements in the SMB. Detail of the SMB stator with 8 YBCO bulks installed in the base.

Both FC and ZFC tests have been performed for the quasi-static measurements. For 
the ZFC tests, the rotor and stator were taken apart $130 \mathrm{~mm}$ before the superconductive material was cooled down below its critical temperature. After cooling down, the magnetic rotor was moved in a quasi-static movement, first approximating rotor and stator up to a gap of $2 \mathrm{~mm}$ and then moving them away to the cooling position. In the sequence, rotor and stator were approximated again to the minimal gap. For the FC measurements, several cooling positions were tested: $27 \mathrm{~mm}, 22 \mathrm{~mm}, 17 \mathrm{~mm}$ and 12 $\mathrm{mm}$. The HTS were cooled in one of the tested cooling position, and the magnetic rotor was approximated until a minimal gap of $2 \mathrm{~mm}$ was reached. After that, the rotor was moved up to $z=130 \mathrm{~mm}$. Then, they were approximated again until reach a minimal gap of $2 \mathrm{~mm}$.

Figure 7 presents the test bench assembled to measure the PMB forces. The procedure applied to the SMB measurements was repeated here for the PMB. Axial and radial displacements were applied to the PMB and the force was measured along both directions. Those measurements define the bearings stability limits and they determine the operational region. The bearing system was designed to allow a lateral and an axial displacements of $5 \mathrm{~mm}$ and $\pm 4 \mathrm{~mm}$, respectively.

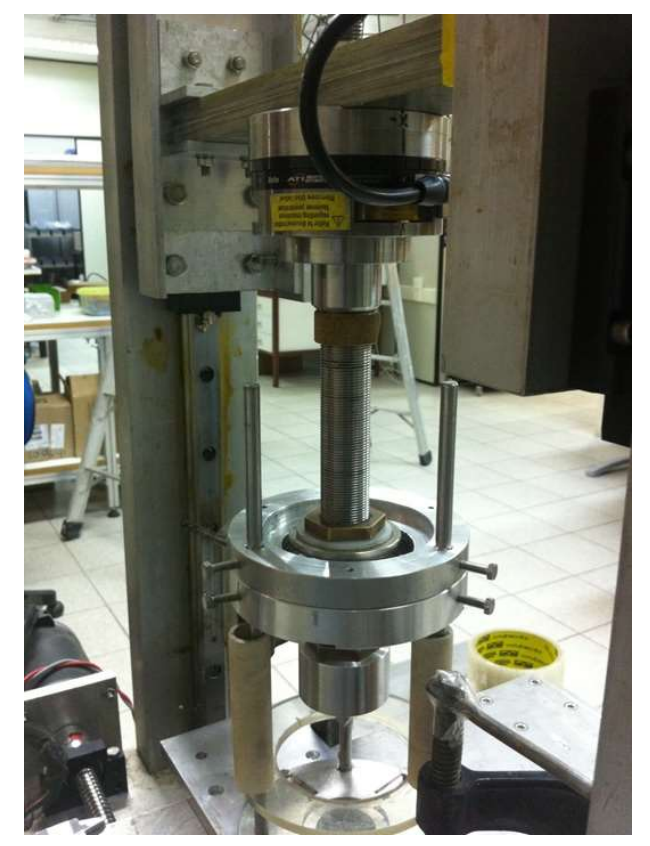

Figure 7. Test bench for the quasi-static measurements in the PMB.

\subsubsection{Dynamic Measurement System}

Another test bench was designed to measure the dynamical behavior of the bearing during its operation for the measurements in the SMB operating without the PMB, as presented in Figure 8. The load cell was positioned in the SMB base, at the SMB static part. For the tests of the SMB operating alone, the external permanent magnet of the PMB is removed, but the internal one is kept into the shaft. However, for the dynamic 
measurements of both magnetic bearings operating together, the PMB is assembled like the detail of Figure 3. The mass of the SMB rotor is $1.183 \mathrm{~kg}$, while the shaft composed by $\mathrm{SMB}$ rotor, PMB rotor and steel axis has $2.750 \mathrm{~kg}$.

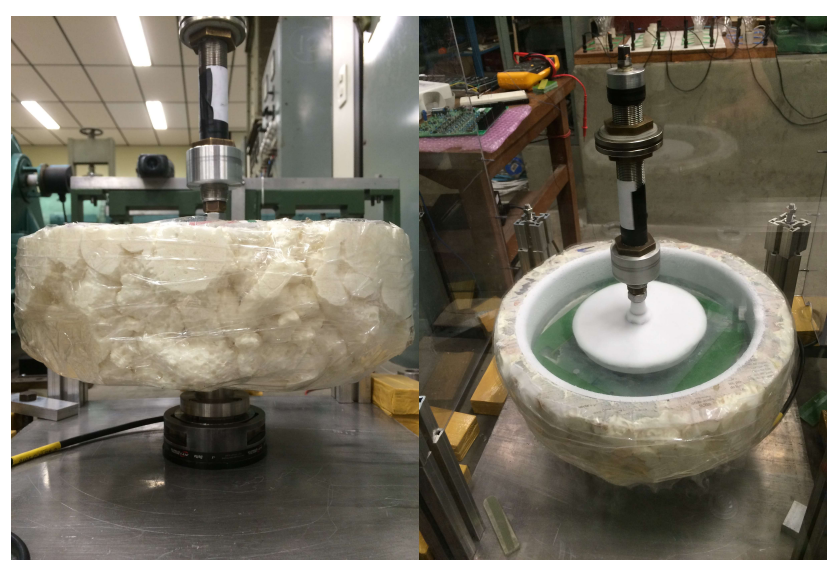

Figure 8. SMB with the vertical shaft during the dynamic measurements.

During the dynamical measurements, an external motor with a flexible axle is temporarily coupled with the shaft, in order to accelerate it. After reach the desired speed, the motor is removed and the system is lett to rotate freely. The speed and the forces in the shaft are measured during the speed down time. This experiment is meant for determining the resonance speeds that should be avoided during the operation of the bearings.

\section{Results and Discussion}

This section presents the quasi-static and dynamic measurements made with the PMB and the SMB operating alone and assembled together.

\subsection{Permanent Magnet Bearing Quasi-Static Measurement}

The PMBs have an intrinsically instability in one direction. The stability in the system is achieved by the operation in combination with the SMB. As the system is assembled vertically, the first measurements presented are the axial forces in order to determine the equilibrium position and the operation region. It has been done by PMB quasi-static measurements along the axial direction. In this system, the rotor has no physical contact with the stator, so it can be moved in both radial and axial directions. The PMB stator was fixed in the test bench (Figure 7). The rotor was first displaced from the stator vertical center position. Besides, a radial displacement with an amplitude of $5 \mathrm{~mm}$ was applied to every $z$ position. During these $z$ and $\rho$ movements, which followed the discrete increment described in section 2.4, the magnetic static forces in each position was acquired. The results for the measured axial restoring forces are presented in Figure 9. 


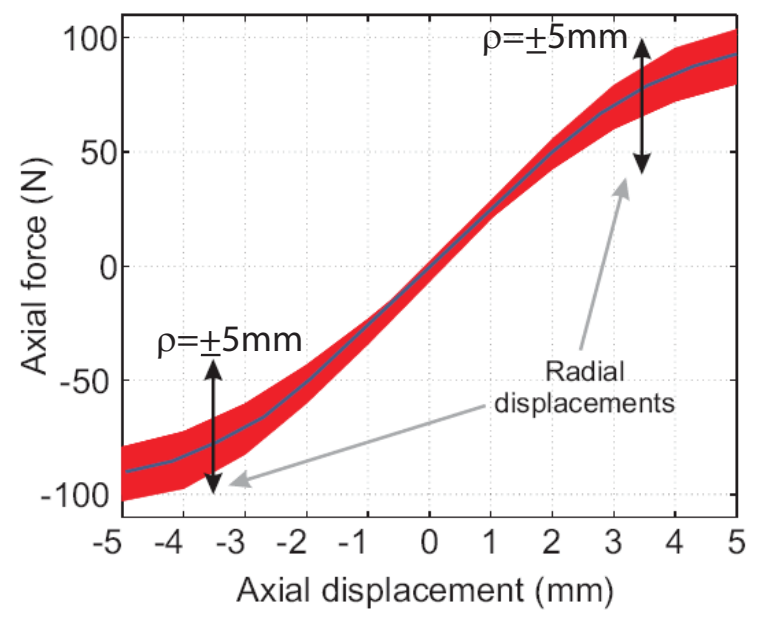

Figure 9. Axial force results for the PMB, with axial displacement and radial perturbation.

Proceeding in the same way, but first displacing the system in steps of $1 \mathrm{~mm}$ in the radial direction, and later applying an axial excursion with $4 \mathrm{~mm}$ amplitude over the $z$ direction to every $\rho$ position, the radial force results were obtained, as presented in Figure 10. It can be observed that displacements along radial direction produces a restoring force to bring the rotor to the central position. The $z$ displacements here trend to reduce the bearing radial stiffness.

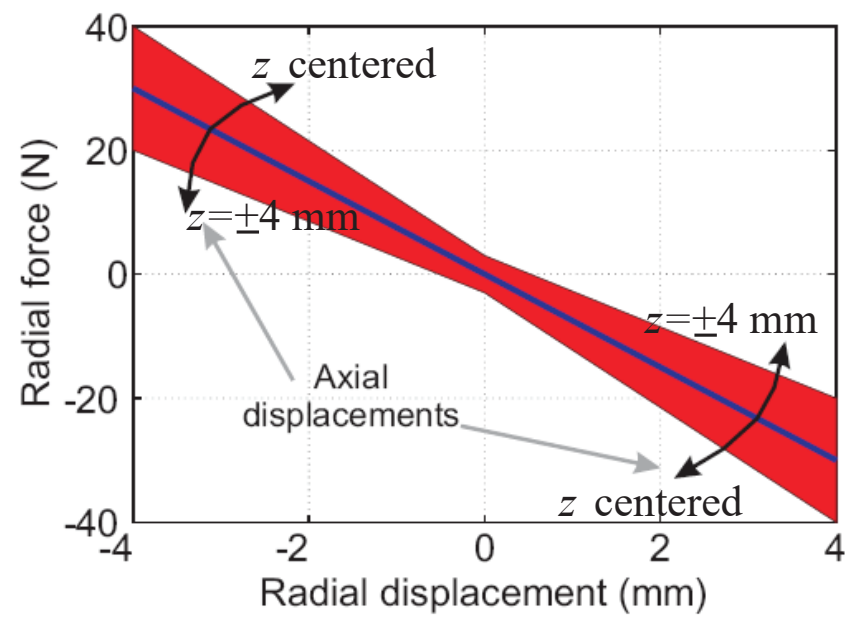

Figure 10. Radial force results for the PMB, with radial displacement and axial perturbation.

\subsection{Superconducting Magnetic Bearing}

\subsubsection{Quasi-static Measurements}


This section presents the quasi-static force measurements for the SMB. Figure 11 shows the measured SMB axial forces during ZFC force measurements, whose experimental procedures were described in section 2.4.1. The hysteresis presented a strait loop, indicating that the HTS bulks used in the experiments have a good quality. Figure 11 shows that the maximum axial force that SMB can produce at a $2 \mathrm{~mm}$ gap is approximately $350 \mathrm{~N}$.

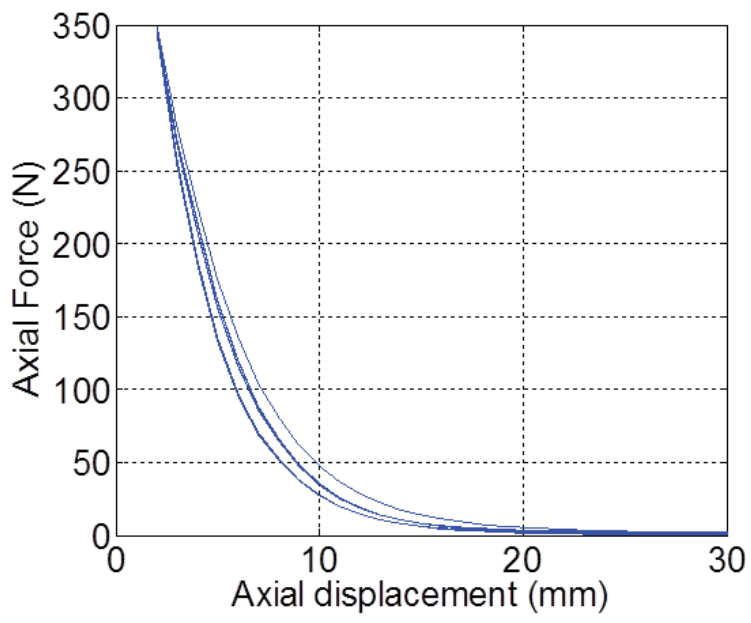

Figure 11. SMB axial force results during ZFC measurements.

The FC axial force results are presented in Figure 12 for the following cooling height $z$ positions between the rotor and the YBCO bulks: 12, 17, 22 and $27 \mathrm{~mm}$. The maximal force at a gap of $2 \mathrm{~mm}$, when a cooling height of $z=27 \mathrm{~mm}$ and $z=12 \mathrm{~mm}$ were made, were respectively, $335 \mathrm{~N}$ and $300 \mathrm{~N}$. Since the maximal decay in the vertical force was $2 \%$, and a smaller cooling height can increase the radial force, it was decided to adopt the cooling height of $12 \mathrm{~mm}$ for the bearing operation.

During a FC process, when the cooling height position is done with the SMB rotor closer to the HTS, more flux is trapped in the superconductor. Two results can be immediately observer in the SMB: smaller axial force and larger radial stiffness. This result will also contribute to determine the cooling and operational positions. In order to determine the radial stiffness, the radial SMB force was measured for a $5 \mathrm{~mm}$ rotor lateral displacement, as presented in Figure 13. The stiffness obtained for the cooling height of $12 \mathrm{~mm}$ and $27 \mathrm{~mm}$ are, $5.2 \mathrm{~N} / \mathrm{mm}$ and $2.6 \mathrm{~N} / \mathrm{mm}$, respectively.

The FC axial force results (Figure 12) combined with the measurements of the axial force in the PMB (Figure 9) will give the information of the operation position determined for the bearing set. By these results, it was determined that the adopted cooling position of the SMB is $12 \mathrm{~mm}$, since the vertical force was reduced only $10 \%$ and the radial stiffness was the double than the $27 \mathrm{~mm}$ cooling height gap.

The SMB and PMB axial forces are superposed in Figure 14. The PMB equilibrium position was displaced from $z=0 \mathrm{~mm}$ to $9 \mathrm{~mm}$. As the SMB is intrinsically stable and the PMB is not, the SMB stiffness magnitude must be slightly higher than the PMB in 


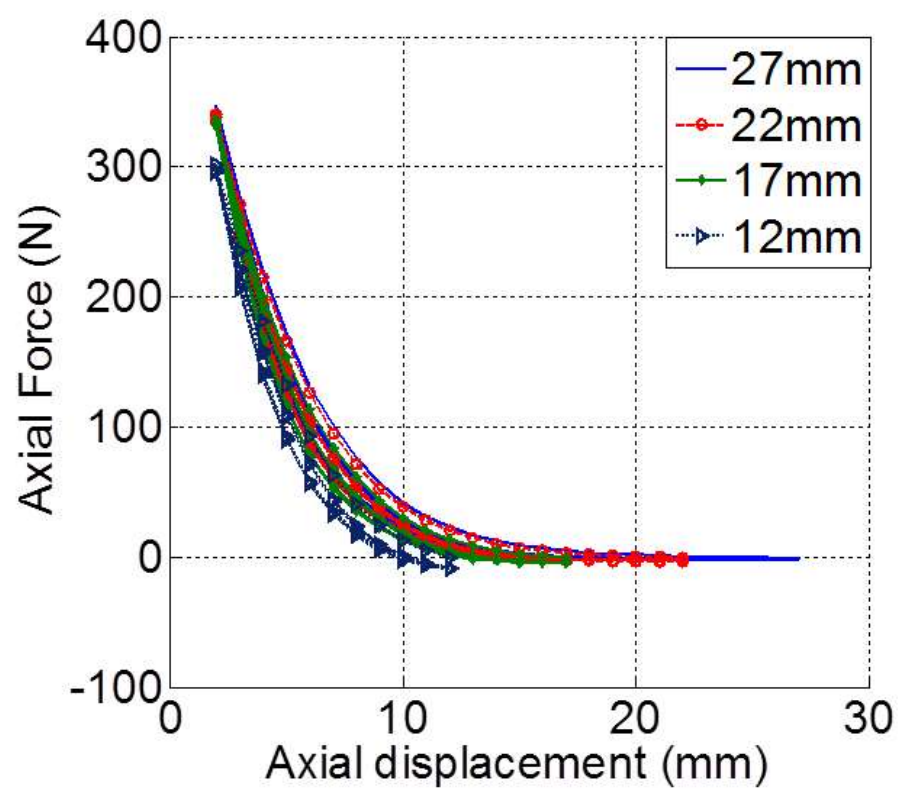

Figure 12. SMB axial force measurements during FC at different cooling height positions.

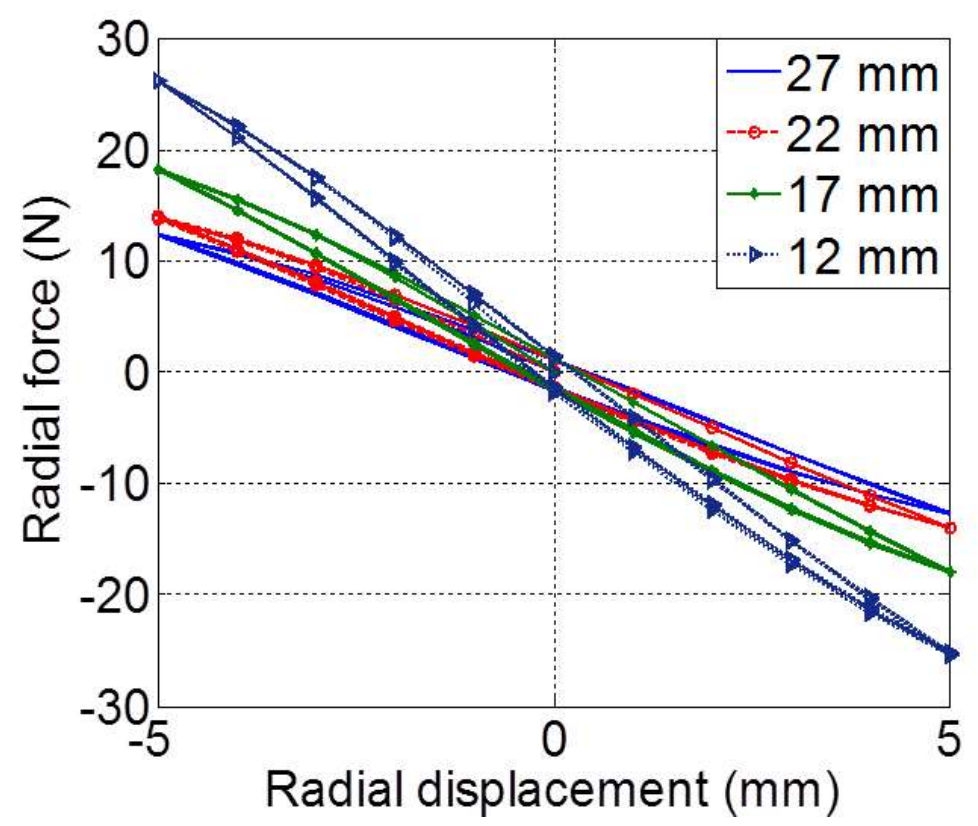

Figure 13. SMB radial force measurements during FC at different cooling height positions.

the operation region. The determined operation point is at $z=7 \mathrm{~mm}$, allowing a \pm 2 mm excursion.

Another configuration with 2 YBCO bulks arranged in the central position of the SMB stator has also been tested. The quasi-static force results are presented in 


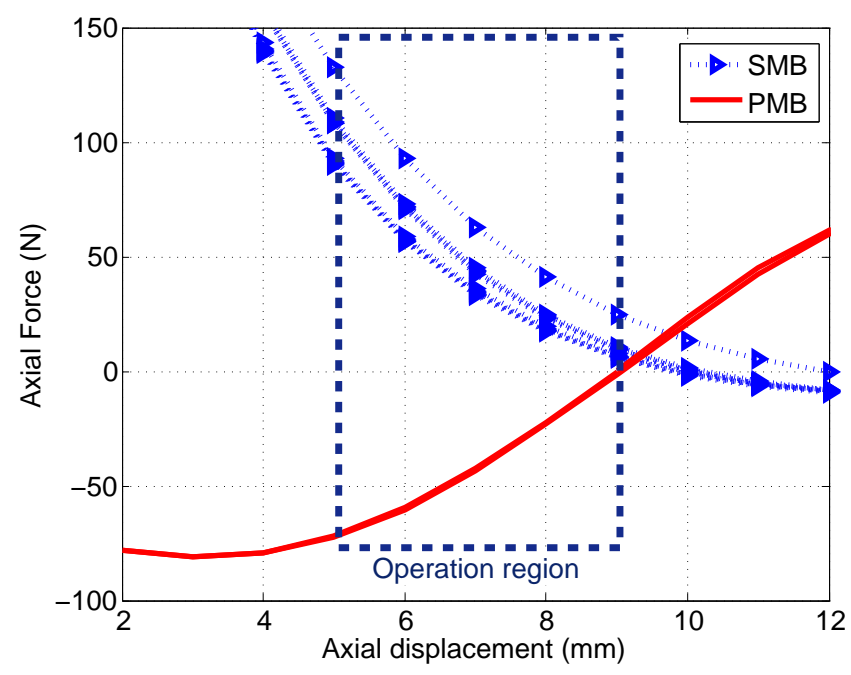

Figure 14. Axial force results of the SMB and the PMB operating together.

Figures 15 and 16 to show the behavior of axial and radial forces interaction due to radial and axial displacements. These results were obtained for a cooling height position of $20 \mathrm{~mm}$. The axial force was measured for different air gaps and different radial displacements. Small radial displacements (in the range of $\pm 10 \mathrm{~mm}$ ) does not have significant influence in the axial force, as can be observed in Figure 15.

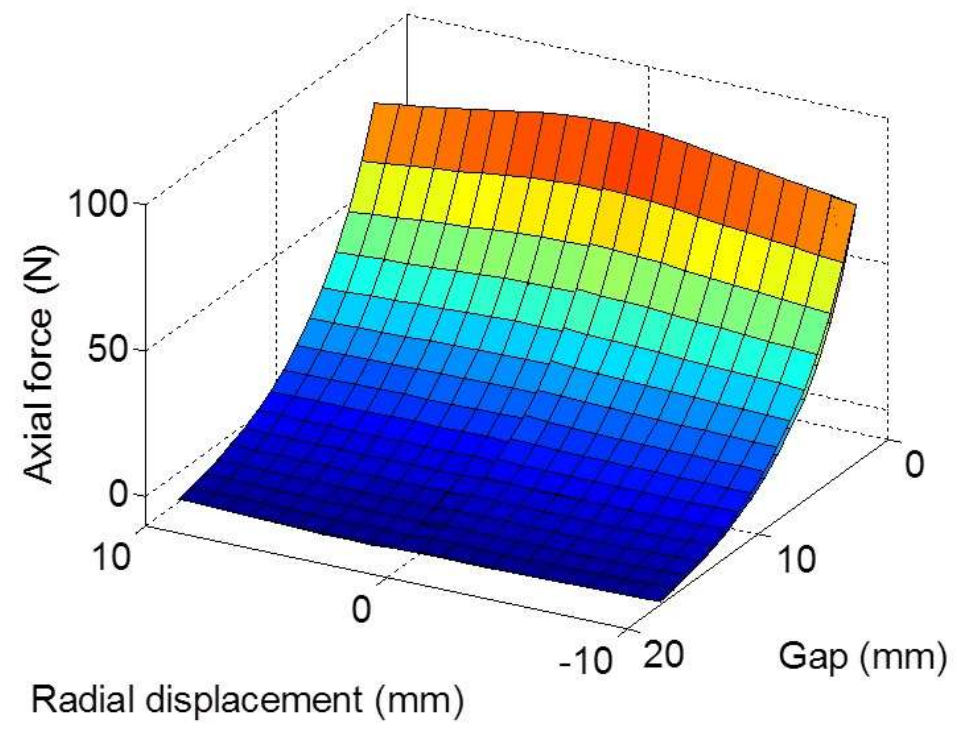

Figure 15. Axial force measured due to axial displacement for different radial displacements. The test was performed using only 2 YBCO bulks in the SMB stator.

Figure 16 shows that the hysteretic effect is smaller for higher gaps, like $20 \mathrm{~mm}$. As the magnetic rotor is moved in the direction of the SMB stator, the magnetic flux start to be pumped inside the YBCO bulks. More field will penetrate in the HTS, resulting 
in an increment of the radial force for smaller radial displacements.

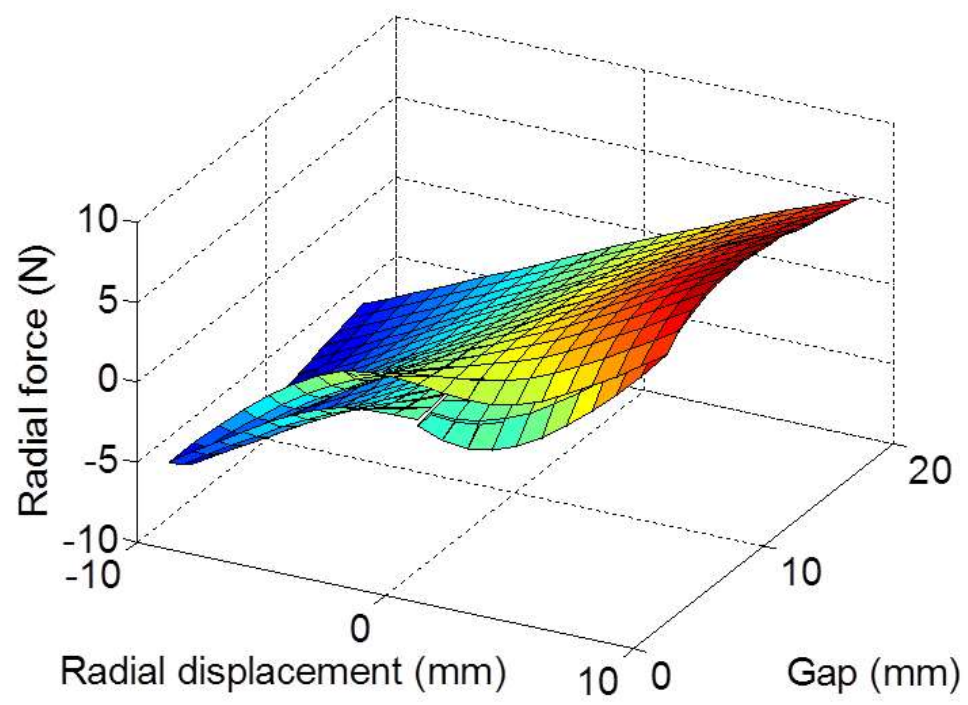

Figure 16. Radial force measured for different axial and radial displacements. The test was performed with only $2 \mathrm{YBCO}$ in the SMB stator.

\subsubsection{Dynamic measurements}

The dynamic measurements were made with the cooling height of $12 \mathrm{~mm}$ for the system presented in Figure 8. In order to do the field cooling, a gap between the superconductors and SMB rotor was initially established by fixing it in the stable position of the PMB. After that, the superconductors are cooled with liquid nitrogen. After the FC process, the rotor was accelerated to the speed of $2650 \mathrm{rpm}$ with the help of an external motor and was released to spin freely. The experiment was made for situations: the SMB operating alone and the SMB + PMB. Figure 17 shows the speed decay along the time for both assembled cases.

The radial and axial measured forces for the same experiment presented in Figure 17 are shown in Figures 18 and 19 for the SMB operating alone and for the SMB + PMB, respectively. Figure 18 (a) presents the force measured in two perpendicular directions $x$ and $y$ for the PMB operating alone, while Figure 18 (b) shows the axial force. In the same way, Figure 19 (a) presents the force measured in the radial direction for the PMB and SMB operating integrated, while Figure 19 (b) presents the axial force results.

The resonance frequencies observed in these results have an important role in the speed decay observed in Figure 17. Between 170 and 180 s, there is a decay in the speed from 1300 to $930 \mathrm{rpm}$ in the PMB + SMB bearing system and a resonance frequency can be clearly observe in Figure 19, by a higher amplitude of the measured forces. For the case of the SMB operating alone, between 720 and $795 \mathrm{~s}$ there is a decay in the speed from 580 to $330 \mathrm{rpm}$, and a higher amplitude of the measured forces were observed in 


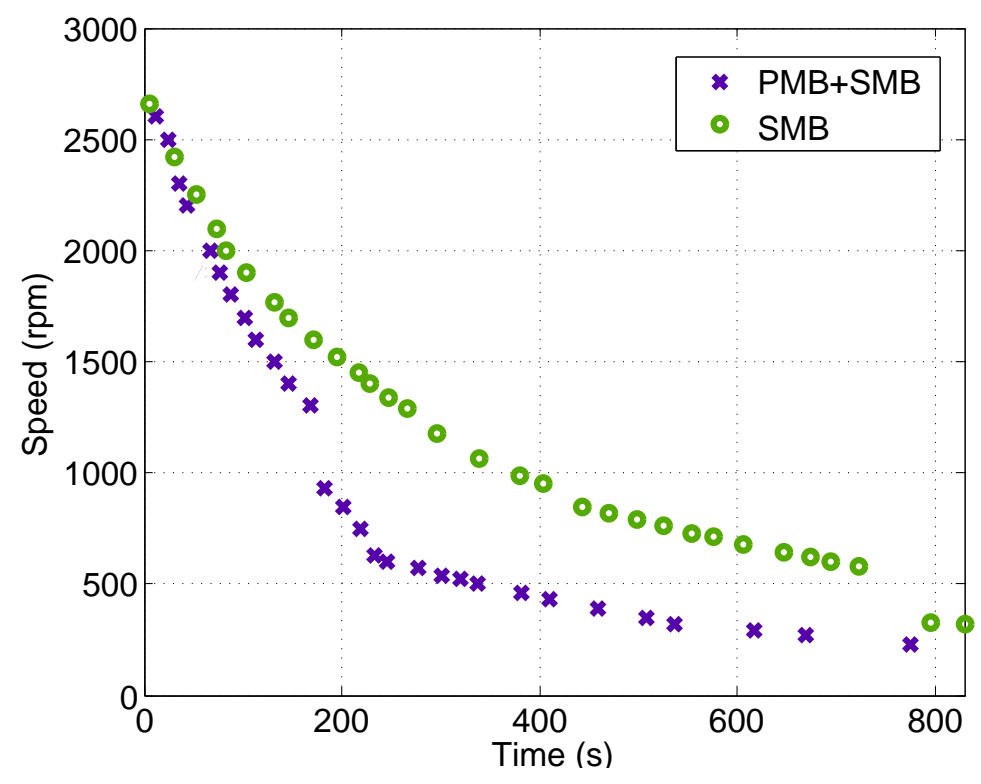

Figure 17. Experimental speed decay results of the SMB operating alone and together with the PMB as a time function.

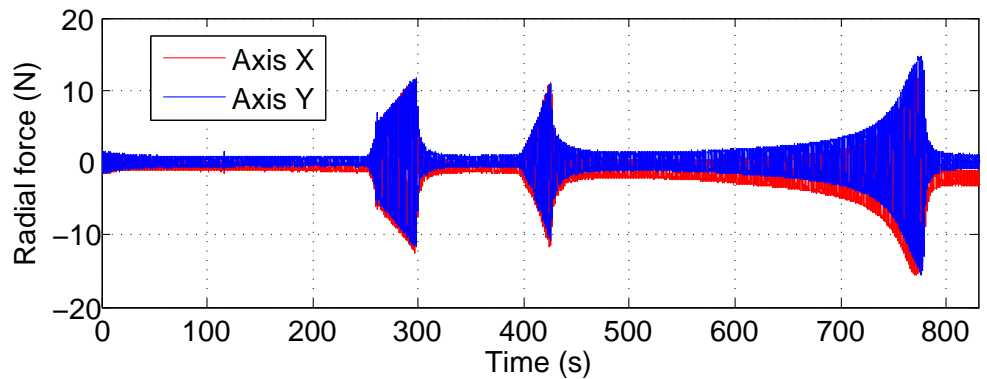

(a)

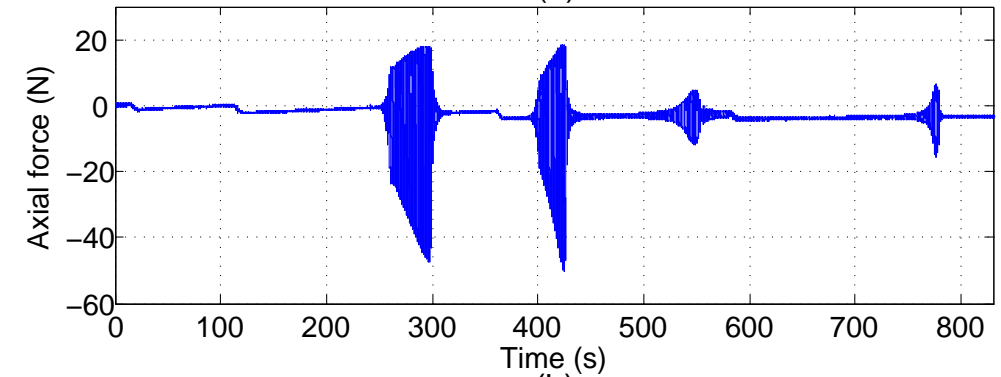

(b)

Figure 18. Measured forces for the SMB operating alone: (a) along the radial direction (b) along the axial direction.

Figure 18.

Figures 18 and 19 show clearly the passage through the resonance frequencies, which produces the speed decay observed in Figure 17. About the SMB operating alone, it presented three resonance speeds ranges, at: 350 to $580 \mathrm{rpm}, 900$ to 950 rpm and 1200 and $1300 \mathrm{rpm}$. Another resonance was observed approximately at 725 


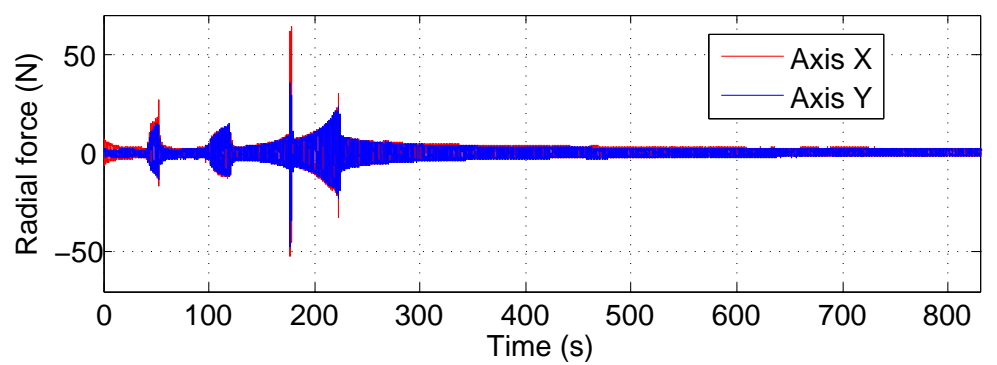

(a)

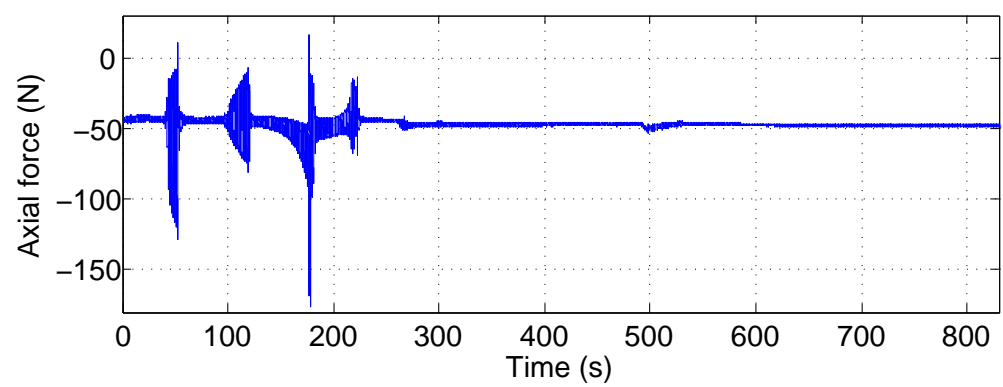

(b)

Figure 19. Measured forces for the SMB and PMB operating integrated: (a) along the radial direction (b) along the axial direction.

rpm. The radial and axial forces of the PMB + SMB show four resonance speeds, at: 660 to $780 \mathrm{rpm}, 930$ to $1300 \mathrm{rpm}, 1600$ to $1700 \mathrm{rpm}$ and 2000 to $2200 \mathrm{rpm}$. As the bearings operating together increased the resonance speed, and the system was tested only in this range, it produced the fast reduction of the speed in the bearing set than for the SMB operating alone. The first explanation considered here for this was the non homogeneity magnetization of the permanent magnets. Hall probe mapping were made in the PM and non perceptive problems in magnetization of the magnets were found. Other possible problem in the PMB is the bad alignment of the permanent magnets. If the axis of the permanent magnets rings used in the PMB are not perfectly aligned, a non homogeneous field would induce currents, producing a continuous decay of the speed, but it does not seem to be the case here. A deeper investigation of the system need to be done for higher speeds.

\section{Conclusions}

A passive magnetic bearing set up was presented, assembled with a Permanent Magnet Bearing (PMB) on the top and a Superconducting Magnetic Bearing (SMB) on the bottom. The bearings have been independently measured for quasi-static results

and operated together for dynamic tests. The results of quasi-static force measurements helped to determine the stable region to be adopted during the operation of the system.

Radial PMB are intrinsically unstable, and here its axial stability was achieved by the operation in combination with the SMB. The PMB has shown radial stability for 
small radial and axial displacements. The PMB presented an axial stiffness of $20 \mathrm{~N} / \mathrm{mm}$ with almost a linear behavior for small axial displacement. The axial force of the SMB increases with almost an exponential rate with the axial position.

For the complete implementation of passive magnetic bearings system, the SMB seems to be a good option, since it presents a similar radial force behavior to that of the bearing set. Moreover, the axial force is reduced but stiffness is bigger that presented by the bearing set.

Dynamic results presented a faster decay of the speed for the PMB+SMB than the one observed for the SMB operating alone. This result was attributed to an increment in the resonance speed of the bearing set. A future investigation need to be done after the introduction of the flywheel and the motor/generator in order to study the new dynamics with the complete system.

\section{Acknowledgments}

The authors would like to thank the financial support received from CNPq, FAPERJ, Swedish Research Council, The Swedish Centre for Smart Grids and Energy Storage (SweGRIDS), STandUP for Energy and CONACYT.

\section{References}

[1] J. Cho, S. Jeong, and Y. Kim. Commercial and research battery technologies for electrical energy storage applications. Progress in Energy and Combustion Science, 48:84-101, 2015.

[2] Nicola Armaroli and Vincenzo Balzani. The hydrogen issue. ChemSusChem, 4(1):21-36, 2011.

[3] Manisha Vangari, Tonya Pryor, and Li Jiang. Supercapacitors: review of materials and fabrication methods. Journal of Energy Engineering, 139(2):72-79, 2012.

[4] M. H. Ali, B. Wu, and R. A. Dougal. An overview of smes applications in power and energy systems. IEEE Transactions on Sustainable Energy, 1(1):38-47, April 2010.

[5] F N Werfel, U Floegel-Delor, R Rothfeld, T Riedel, B Goebel, D Wippich, and P Schirrmeister. Superconductor bearings, flywheels and transportation. Superconductor Science and Technology, 25(1):014007, 2012.

[6] Atul Sharma, V.V. Tyagi, C.R. Chen, and D. Buddhi. Review on thermal energy storage with phase change materials and applications. Renewable and Sustainable Energy Reviews, 13(2):318 $-345,2009$.

[7] R. Saidur, N.A. Rahim, and M. Hasanuzzaman. A review on compressed-air energy use and energy savings. Renewable and Sustainable Energy Reviews, 14(4):1135 - 1153, 2010.

[8] J.P. Deane, B.P. Gallachir, and E.J. McKeogh. Techno-economic review of existing and new pumped hydro energy storage plant. Renewable and Sustainable Energy Reviews, 14(4):1293 $1302,2010$.

[9] T.M.I. Mahlia, T.J. Saktisahdan, A. Jannifar, M.H. Hasan, and H.S.C. Matseelar. A review of available methods and development on energy storage; technology update. Renewable and Sustainable Energy Reviews, 33:532-545, 2014.

[10] Sam Koohi-Kamali, V.V. Tyagi, N.A. Rahim, N.L. Panwar, and H. Mokhlis. Emergence of energy storage technologies as the solution for reliable operation of smart power systems: A review. Renewable and Sustainable Energy Reviews, 25:135 - 165, 2013.

[11] S. Vazquez, S. M. Lukic, E. Galvan, L. G. Franquelo, and J. M. Carrasco. Energy storage systems 
for transport and grid applications. IEEE Transactions on Industrial Electronics, 57(12):38813895, Dec 2010.

[12] J. Cibulka. Kinetic energy recovery system by means of flywheel energy storage. Advanced Engineering, 3(1):27-38, 2009.

[13] A. A. Boretti. Improvements of vehicle fuel economy using mechanical regenerative braking. International Journal of Vehicle Design, 55(1):35-48, 2011.

[14] Rubens De Andrade, Guilherme G Sotelo, Antonio C Ferreira, Luis GB Rolim, José L da Silva Neto, Richard M Stephan, Walter I Suemitsu, and Roberto Nicolsky. Flywheel energy storage system description and tests. Applied Superconductivity, IEEE Transactions on, 17(2):2154-2157, 2007.

[15] Juan Manuel Carrasco, Leopoldo Garcia Franquelo, Jan T Bialasiewicz, Eduardo Galván, Ramón C Portillo Guisado, Ma Ángeles Martín Prats, José Ignacio León, and Narciso Moreno-Alfonso. Power-electronic systems for the grid integration of renewable energy sources: A survey. Industrial Electronics, IEEE Transactions on, 53(4):1002-1016, 2006.

[16] Y. Feng, H. Lin, J. Yan, and Y. Huang. Loss component analysis of flywheel energy storage system with mechanical bearings. Dongnan Daxue Xuebao (Ziran Kexue Ban)/Journal of Southeast University (Natural Science Edition), 43(1):71-75, 2013. cited By 0.

[17] S. Jiang, H. Wang, and S. Wen. Flywheel energy storage system with a permanent magnet bearing and a pair of hybrid ceramic ball bearings. Journal of Mechanical Science and Technology, 28(12):5043-5053, 2014.

[18] M. A. Pichot, J. P. Kajs, B. R. Murphy, A. Ouroua, B. M. Rech, R. J. Hayes, J. H. Beno, G. D. Buckner, and A. B. Palazzolo. Active magnetic bearings for energy storage systems for combat vehicles. IEEE Transactions on Magnetics, 37(1):318-323, Jan 2001.

[19] Y.H. Han, B.J. Park, S.Y. Jung, and S.C. Han. Study of superconductor bearings for a 35 kwh superconductor flywheel energy storage system. Physica C: Superconductivity, 483:156 - 161, 2012.

[20] K. Murakami, M. Komori, H. Mitsuda, and A. Inoue. Design of an energy storage flywheel system using permanent magnet bearing (pmb) and superconducting magnetic bearing (smb). Cryogenics, 47(4):272 - 277, 2007.

[21] H. Mitsuda, A. Inoue, B. Nakaya, and M. Komori. Improvement of energy storage flywheel system with smb and pmb and its performances. IEEE Transactions on Applied Superconductivity, 19(3):2091-2094, June 2009.

[22] M. Subkhan and M. Komori. New concept for flywheel energy storage system using smb and pmb. IEEE Transactions on Applied Superconductivity, 21(3):1485-1488, June 2011.

[23] G.G. Sotelo, D.H.N. Dias, R. De Andrade Jr., and R.M. Stephan. Tests on a superconductor linear magnetic bearing of a full-scale maglev vehicle. IEEE Transactions on Applied Superconductivity, 21(3 PART 2):1464-1468, 2011. cited By 25.

[24] G. G. Sotelo, A. C. Ferreira, and R. de Andrade. Halbach array superconducting magnetic bearing for a flywheel energy storage system. IEEE Transactions on Applied Superconductivity, 15(2):2253-2256, June 2005.

[25] G. G. Sotelo, R. de Andrade, and A. C. Ferreira. Magnetic bearing sets for a flywheel system. IEEE Transactions on Applied Superconductivity, 17(2):2150-2153, June 2007.

[26] Elkin Rodriguez, Juan de Santiago, Jesus José Pérez-Loya, Felipe S Costa, Guilherme G Sotelo, Janaina Goncalves de Oliveira, and Richard M Stephan. Analysis of passive magnetic bearings for kinetic energy storage systems. In 14th International Symposium on Magnetic Bearings, 2014.

[27] Elkin Rodriguez, Jesus José Pérez-Loya, Juan de Santiago, Felipe S Costa, Guilherme G Sotelo, Janaína G Oliveira, and Richard M Stephan. Passive magnetic bearing system. In 22nd International Conference on Magnetically Levitaded Systems and Linear Drives, 2014. 